\title{
ARMAZENAMENTO DE CAQUI (Diospyros kaki, L.) cv. QUIOTO, EM ATMOSFERA CONTROLADA ${ }^{1}$
}

\author{
JOEL DONAZZOLO² \& AURI BRACKMANN³
}

\begin{abstract}
RESUMO - Objetivou-se avaliar, com o presente trabalho, o efeito da temperatura e do $\mathrm{CO}_{2}$ no armazenamento em atmosfera controlada sobre a conservação de caqui cv. Quioto. Foram avaliadas as temperaturas $-1,0 \mathrm{e}-0,5^{\circ} \mathrm{C}$, e pressões parciais de $\mathrm{CO}_{2}$ de $0 ; 5$ e $10 \mathrm{kPa}$, com os tratamentos arranjados em um esquema bifatorial. Os frutos foram avaliados após 3 meses de armazenamento mais 3 dias de exposição à temperatura ambiente $\left(18-20^{\circ} \mathrm{C}\right)$. Conforme os resultados, não foi constatada interação entre os fatores, havendo efeito significativo para temperatura somente na firmeza de polpa, em que $-1,0^{\circ} \mathrm{C}$ apresentou frutos mais firmes. As diferentes pressões parciais de $\mathrm{CO}_{2}$ não influenciaram a perda de peso e a firmeza de polpa. As podridões apresentaram uma resposta linear negativa em relação ao $\mathrm{CO}_{2}$, porém, mantendo elevada ocorrência. Valores de $\mathrm{CO}_{2}$ entre 5 e 10kPa proporcionaram frutos com menor índice de escurecimento de epiderme e com coloração mais amarela e vermelha. $\mathrm{O} \mathrm{CO}_{2}$ entre 5 e $10 \mathrm{kPa}$ apresentou os melhores resultados, que somados à temperatura de $-1,0^{\circ} \mathrm{C}$, foi a melhor condição de armazenamento, que, no entanto, teve o período de conservação inferior a três meses nesta condição, devido às altas perdas por podridões.
\end{abstract}

Termos para indexação: temperatura, $\mathrm{CO}_{2}$, conservação

\section{CONTROLLED ATMOSPHERE STORAGE OF QUIOTO PERSIMMONS (Diospyros kaki, L.)}

\begin{abstract}
The present work had the objective to evaluate the effect of the temperature and $\mathrm{CO}_{2}$ in controlled atmosphere storage on the quality of 'Quioto' persimmons. Temperatures of $-1,0$ and $-0,5^{\circ} \mathrm{C}$ and $\mathrm{CO}_{2}$ partial pressures of 0,5 and $10 \mathrm{kPa}$ were evaluated, and treatments were organized in a bifatorial design. The fruits were evaluated after 3 months of storage more 3 days of shelf-life $\left(18-20^{\circ} \mathrm{C}\right)$. There was only significant effect of the temperature on pulp firmness. The Fruits stored at $-1,0^{\circ} \mathrm{C}$ showed higher pulp firmness than fruits at $-0,5^{\circ} \mathrm{C}$. Different $\mathrm{CO}_{2}$ partial pressures did not have influence on the weight loss and pulp firmness. A negative lineal response was observed for decay in relation to $\mathrm{CO}_{2}$ partial pressures, which incidence was very high. The other parameters had a quadratic response. In CA storage with $\mathrm{CO}_{2}$ value between 5 and $10 \mathrm{kPa}$ the fruits had a lower skin browning and more yellow and red coloration. The $\mathrm{CO}_{2}$ between 5 and $10 \mathrm{kPa}$ presented the best results and added to temperature of $-1,0^{\circ} \mathrm{C}$ were the best storage condition, however, the storage period should be shorter than 3 months at these condition, due to high losses with decay.
\end{abstract}

Index terms: temperature, $\mathrm{CO}_{2}$, conservation

\section{INTRODUÇÃO}

O caqui- 'Quioto' vem despertando interesse dos produtores devido à sua colheita tardia, depois da cv. Fuyu, além de ser bem aceito pelo consumidor, por apresentar polpa com cor "chocolate" não taninosa. Seus frutos são redondo-alongados de bom tamanho, pertencentes ao grupo variável com altos índices de autopolinização, o que lhe confere a característica de apresentar sementes. Poucos estudos têm sido feitos com essa cultivar, principalmente com relação ao seu potencial de armazenamento.

O caqui foi introduzido no Brasil por volta de 1890, em São Paulo. Hoje, as regiões Sul e Sudeste são as principais produtoras, por apresentarem condições edafoclimáticas adequadas (Martins \& Pereira, 1989). No RS, a área da cultura vem crescendo nos últimos anos por ser de fácil manejo, pouco uso de agrotóxicos e boa aceitação no mercado.

O baixo consumo per capita no Brasil, o curto período de colheita, a alta perecibilidade dos frutos e o mercado regionalizado fazem com que a oferta no período de safra seja muito maior que a demanda, ocasionando queda de preços, perdas das mais diversas, desde aquela parte da produção que não é colhida por falta de mercado, até aquela desperdiçada no sistema de comercialização, com um conseqüente desabastecimento do mercado por 7 a 8 meses do ano pela falta do produto. Seu armazenamento poderia contribuir para contornar estes problemas.

Segundo Brackmann \& Donazzolo (2000), a conservação do caqui depende principalmente das condições de armazenamento. No entanto, os fatores pré-colheita, como sanidade do pomar, nutrição mineral e ponto de maturação não são menos importantes. De acordo com estes autores, as condições de temperatura e umidade relativa (UR) das câmaras são os fatores mais importantes no armazenamento, e o uso de atmosfera controlada (AC), modificada (AM) e absorção de etileno podem melhorar a qualidade dos frutos.

1 (Trabalho 169/2001). Recebido: 17/10/2001. Aceito para publicação: 02/05/2002. Parte do trabalho do $1^{\circ}$ autor, para obtenção do grau de Mestre em Agronomia, Programa de Pós-graduação em Agronomia, da Universidade Federal de Santa Maria, Santa Maria - RS.

2 Eng ${ }^{\circ}$ Agroํ, Msc., Extencionista da ASCAR/EMATER-RS, (54)361-1089, donazzolo@ bol.com.br.

3 Eng ${ }^{\mathrm{o}}$ Agr ${ }^{\mathrm{o}}$, Doutor, Professor do Departamento de Fitotecnia, Centro de Ciências Rurais (CCR), UFSM, 97015-900, Santa Maria - RS, (55) 2208179, brackman@ccr.ufsm.br. 
A temperatura ideal para a conservação de caqui ainda não está definida. No entanto, $0^{\circ} \mathrm{C}$ é a mais recomendada para uma boa conservação, diminuindo a intensidade de degradação dos ácidos e açúcares e retardando a senescência (Lee et al., 1993; Brackmann et al., 1997). Para a cv. Triumph, Prusky et al. (1997) utilizaram $-1^{\circ} \mathrm{C}$ e Brackmann \& Saquet (1995) verificaram que $-0,5^{\circ} \mathrm{C}$ foi melhor que $0,5^{\circ} \mathrm{C}$ para a cv. Fuyu.

Aliada à baixa temperatura, pode-se utilizar a $\mathrm{AC}$ para retardar a perda de qualidade durante o armazenamento. As altas concentrações de $\mathrm{CO}_{2}$ inibem diversas enzimas do ciclo dos ácidos tricarboxílicos, reduzindo o metabolismo e a respiração dos frutos (Brackmann \& Chitarra, 1998). Não se dispõe de informações precisas acerca das concentrações de gases para armazenar caqui em AC. Pressões parciais de $16 \mathrm{kPaO}_{2} / 15 \mathrm{kPaCO}_{2}$ são citadas por Brackmann et al. (1997) como boas condições de armazenamento para a cv. Fuyu, e $8 \mathrm{kPaCO}_{2} / 2 \mathrm{kPaO}_{2} \mathrm{em}-0,5^{\circ} \mathrm{C}$ são tidas como boas condições de armazenamento para as cultivares Taubaté, Bauru e Fuyu (Brackmann \& Saquet, 1995).

O objetivo do presente trabalho foi avaliar o efeito de condições de baixas temperaturas e de altas pressões parciais de $\mathrm{CO}_{2}$ no armazenamento, em AC, sobre a qualidade dos frutos de caqui cv. Quioto, visando a diminuir perdas em pós-colheita e prolongar o período de conservação.

\section{MATERIAL E MÉTODOS}

Os frutos utilizados foram da cv. Quioto, provenientes de um pomar comercial de Farroupilha - RS, colhidos com a coloração da epiderme laranja-amarelada, ponto de maturação considerado adequado para a conservação por longo período, e firmeza de polpa acima de 50N. Após a colheita, os frutos foram transportados ao Núcleo de Pesquisa em Pós-Colheita (NPP) do Departamento de Fitotecnia da Universidade Federal de Santa Maria, onde foram submetidos a um processo de seleção, excluindo-se aqueles que apresentavam maturação avançada, baixo calibre ou com ferimentos. Posteriormente, foi efetuada uma homogeneização das amostras experimentais e aplicados os tratamentos. O delineamento experimental foi o inteiramente casualizado, arranjado em um esquema bifatorial $2 \times 3$, com quatro repetições por tratamento e unidade experimental composta por 19 frutos.

Os tratamentos constaram de uma combinação de duas temperaturas $\left(-1,0 \mathrm{e}-0,5^{\circ} \mathrm{C}\right.$, com uma oscilação de $\left.\pm 0,2^{\circ} \mathrm{C}\right)$ e três pressões parciais de $\mathrm{CO}_{2}(0 ; 5$ e $10 \mathrm{kPa})$. A umidade relativa do ar foi mantida acima de $95 \%$ e o $\mathrm{O}_{2}$ acima de $15 \mathrm{kPa}$.

Os frutos foram armazenados em minicâmaras experimentais herméticas, com capacidade de 72L, mantidas, durante o período de armazenamento, em duas câmaras frigoríficas de $24 \mathrm{~m}^{3}$, onde se estabeleceu o controle automático da temperatura. Os teores de $\mathrm{CO}_{2}$ foram obtidos mediante injeção deste gás nas minicâmaras. $\mathrm{O}$ processo respiratório dos frutos resultou no consumo de $\mathrm{O}_{2}$ e acúmulo de $\mathrm{CO}_{2}$, e, para manutenção dos níveis estabelecidos, foram realizadas diariamente análises e correções. A determinação das concentrações dos gases foi feita através de analisadores eletrônicos de $\mathrm{O}_{2}$ e $\mathrm{CO}_{2}$ marca Agridatalog, de fluxo contínuo, pelos quais se circulou uma amostra de gás de cada minicâmara que, após a análise, retornou à respectiva minicâmara. As leituras foram feitas em porcentagem que, na altitude de Santa Maria, praticamente equivale à unidade $\mathrm{kPa} . \mathrm{O}$ $\mathrm{O}_{2}$ consumido pela respiração dos frutos foi compensado pela injeção de ar nas minicâmaras. $\mathrm{O} \mathrm{CO}_{2}$, quando em excesso, foi eliminado, circulando-se o gás das minicâmaras por uma solução absorvente com hidróxido de potássio a $40 \%$.

A concentração do etileno nas minicâmaras de atmosfera controlada foi monitorada semanalmente durante todo o período de armazenamento. Uma seringa com volume de $50 \mathrm{ml}$ era enchida com a atmosfera homogeneizada de cada minicâmara e desta eram injetadas duas amostras de $1 \mathrm{ml}$ em um cromatógrafo a gás, equipado com uma coluna Porapak N 80/100 e detector de ionização de chama (FID).

Na instalação do experimento, foi realizada uma análise de qualidade de três amostras de 10 frutos. As avaliações subsequientes foram realizadas após três meses de armazenamento mais três dias de exposição à temperatura ambiente $\left(18-20^{\circ} \mathrm{C}\right)$, simulando o período de comercialização. Os parâmetros de qualidade avaliados foram firmeza de polpa $(\mathrm{N})$ e incidência de podridões (\%), conforme descrito por Brackmann et al. (1997); índice de escurecimento da epiderme, determinado através de níveis de manifestação, onde o nível 1 corresponde ao fruto com área menor de $10 \%$ da epiderme com escurecimento; 2 com epiderme do fruto apresentando entre 10 e $30 \%$ de escurecimento; e 3 com escurecimento maior de $30 \%$. O índice foi calculado pela soma dos produtos do número de frutos pelo seu nível, dividido pelo número total de frutos da amostra; perda de peso $(\%)$, calculada pela diferença de peso da amostra por ocasião da entrada na câmara e da data de avaliação do experimento; sólidos solúveis totais $\left({ }^{\circ} \mathrm{B}\right)$, determinados por refratometria, utilizando o suco de uma fatia mediana dos frutos de cada amostra; cor da epiderme obtido por um colorímetro eletrônico, da marca Minolta, que utiliza o sistema CIE L*, a*, $\mathrm{b}^{*}$; e, respiração $\left(\mathrm{mLCO}_{2} \cdot \mathrm{kg}^{-1} \cdot \mathrm{h}^{-1}\right)$ e produção de etileno $\left(\mathrm{mLC}_{2} \mathrm{H}_{4} \cdot \mathrm{kg}^{-1} \cdot \mathrm{h}^{-1}\right)$, determinados através da quantificação do $\mathrm{CO}_{2}$ e do etileno produzido por 10 frutos hermeticamente fechados em um recipiente de vidro com capacidade de 5 litros, durante aproximadamente 2 horas. Esta análise foi realizada 24 e 48 horas após a saída dos frutos da câmara, no período de simulação da comercialização e contou com apenas duas repetições, não sendo, por este motivo, submetidas à análise estatística.

Para cada parâmetro avaliado, foi efetuada uma análise da variância, sendo as médias do fator temperatura comparadas estatisticamente pelo teste $\mathrm{F}$, ao nível de $5 \%$ de probabilidade do erro e o fator $\mathrm{CO}_{2}$ submetido a uma análise de regressão. As variáveis perda de peso e podridões, expressas em percentagem, foram transformadas pela fórmula $\sqrt{x}$, e arc-sen $\sqrt{\mathrm{x} / 100}$, respectivamente, antes da análise da variância.

\section{RESULTADOS E DISCUSSÃO}

A interação entre os fatores (temperatura e $\mathrm{CO}_{2}$ ) não foi encontrada para nenhum dos parâmetros avaliados (Tabela 1). Isso indica que a resposta de cada fator dentro dos níveis do outro fator foi semelhante. Possivelmente, o intervalo muito pequeno entre as temperaturas utilizadas levou a esse resultado, tanto que foi constatada diferença significativa entre as duas temperaturas apenas para a variável firmeza de polpa (Tabela 2).

Isoladamente, o $\mathrm{CO}_{2}$ não se mostrou influente na redu- 
ção da perda de peso (Figura 1B) e da firmeza de polpa (Figura 1A ), ao passo que as podridões apresentaram uma resposta linear negativa (Figura 1A). Para os parâmetros, SST, cor e índice de escurecimento de epiderme, a resposta foi quadrática, sendo que valores de $\mathrm{CO}_{2}$ entre 5 e $10 \mathrm{kPa}$ apresentaram os melhores resultados (Figuras 1B e 1C).

Com relação à cor da epiderme, os frutos em alto teor de $\mathrm{CO}_{2}$ apresentaram coloração mais amarela $\left(b^{*}\right)$ e mais vermelha $\left(\mathrm{a}^{*}\right)^{2}$ (Figura $1 \mathrm{C}$ ), representando que o $\mathrm{CO}_{2}$ acelerou os processos de mudança da cor de epiderme. Isto confirma que os pigmentos no caqui continuam a ser sintetizados durante o armazenamento, conforme observado por Turk (1993), quando da aplicação de 0,25 a $0,5 \mathrm{kPa}$ de $\mathrm{CO}_{2}$ por 46 horas após o armazenamento, o que estimulou a síntese de caroteno, fazendo parte das transformações que ocorrem no amadurecimento. Como altas concentrações de $\mathrm{CO}_{2}$ são indicadas para estimular a destanização do caqui (Guelfat-Reich et al, 1975), significa que este gás tem efeito acelerador de alguns processos do amadurecimento. A variação da pressão parcial do $\mathrm{O}_{2} \mathrm{e}$, principalmente, do $\mathrm{CO}_{2}$, pode estimular a síntese de a-caroteno (Wright \& Kader, 1997), o que explica o aumento da coloração amarela. A coloração vermelha mais intensa no caqui é uma característica desejável, desde que não venha acompanhada da perda de firmeza de polpa. Os teores de $\mathrm{CO}_{2}$ estudados neste trabalho parecem apresentar este efeito, visto que produziram frutos mais vermelhos e não influenciaram a firmeza de polpa (Figuras 1A e 1C).

Com pressões parciais de $\mathrm{CO}_{2}$ próximas a $10 \mathrm{kPa}$ constataram-se os menores índices de escurecimento da epiderme (Figura 1B), que é a principal causa de perda do caqui armazenado. Lee et al.(1993), Brackmann et al. (1997) e Park (1997) confirmam estes resultados. Isso sinaliza que esse dano pode estar relacionado à senescência dos frutos, ou, mais propriamente, ao processo respiratório, já que esse fator $\left(\mathrm{CO}_{2}\right)$ tem ação inibitória marcante na cadeia respiratória (Brackmann \& Chitarra, 1998). A análise dos valores de produção de etileno e respiração dos frutos após a saída da câmara (Figura 1D) reforça esta hipótese. A respiração dos frutos mantidos a $0 \mathrm{kPa}$ de $\mathrm{CO}_{2}$ foi maior do que a dos frutos armazenados a 10kPa e, especialmente, aqueles mantidos a $5 \mathrm{kPa}$. Verificou-se que, após um dia de permanência a 18$20^{\circ} \mathrm{C}$, depois de 3 meses de armazenamento com $10 \mathrm{kPa}$ de $\mathrm{CO}_{2}, \mathrm{a}$ produção de etileno foi zero, e em $5 \mathrm{kPa}$ foi aproximadamente a metade do que produziram os frutos mantidos em $0 \mathrm{kPa}$. No segundo dia, os frutos mantidos a $0 \mathrm{kPa}$ de $\mathrm{CO}_{2}$, durante o armazenamento, produziram três vezes mais etileno que aqueles mantidos a 5 ou $10 \mathrm{kPa}$, confirmando que altas pressões parciais de $\mathrm{CO}_{2}$ inibem a evolução da produção do etileno (Kader, 1986).
Esta maior produção de etileno provavelmente acelerou os processos metabólicos relacionados à senescência, evidenciados pelos dados de respiração, e estimulou a manifestação do escurecimento de epiderme. A absorção do etileno contribui para a redução da incidência deste distúrbio (Lee, et al., 1993), que pode estar relacionado à maturação avançada e à oxidação de polifenóis dos frutos (Kimet al., 1992; Ben-Arie \& Zutkhi ,1992), devido à alta atividade da enzima polifenol oxidase, que é maior em frutos com elevada incidência do distúrbio (Park, 1997). Já que a aplicação de antioxidante $\left(2.000 \mathrm{mg} . \mathrm{L}^{-1}\right.$ de difenilamina ou $2.500 \mathrm{mg} . \mathrm{L}^{-1}$ de etoxiquin) contribui para a redução da incidência do escurecimento (Lee, et al., 1993), é realmente possível que um processo de oxidação esteja envolvido (Burmeister et al., 1997). Apesar de o fator $\mathrm{CO}_{2}$ apresentar efeito positivo, o índice de escurecimento de epiderme manteve-se elevado (aproximadamente 1,7 - equivale a $\pm 20 \%$ da superfície escurecida), mostrando a necessidade de se investigar outras técnicas para a redução da incidência do distúrbio. Também há a possibilidade de as manchas escurecidas serem causadas pela ocorrência de fungos ( $\mathrm{Al}$ ternaria alternata), como observado por Ben-Arie \& Zutkhi (1992). Entretanto, as manchas não apresentavam características evidentes de ataque de patógenos.

A firmeza de polpa não apresentou resposta às diferentes pressões parciais de $\mathrm{CO}_{2}$ (Figura 1A). Em outras cultivares de caqui, no entanto, condições de AC retardaram a perda de firmeza de polpa (Brackmann et al., 1997; 1999; Park, 1997). Embora o alto teor de $\mathrm{CO}_{2}$ tenha reduzido a respiração e produção de etileno após a saída da câmara (Figura 1D), o índice de escurecimento de epiderme e as podridões (Figuras 1A e 1B), não deve ter afetado a degradação das pectinas e hemicelulose da parede celular, tidas como responsáveis pela firmeza de polpa. No entanto, foi o único parâmetro a apresentar diferença com relação à temperatura. A atividade das enzimas envolvidas no processo de perda de firmeza de polpa parece apresentar diferenças marcantes para o caqui em pequenas variações de temperatura, quando esta se aproxima da ideal, que para a cv. Quioto está, provavelmente, abaixo de $-0,5^{\circ} \mathrm{C}$.

$\mathrm{O}$ fator $\mathrm{CO}_{2}$ apresentou uma relação direta na redução da incidência de podridões (Figura 1A). Prusky et al. (1997) também encontraram resultados semelhantes. Provavelmente por influenciar diretamente na germinação dos esporos e desenvolvimento do fungo e, indiretamente, por retardar o processo metabólico de senescência dos frutos armazenados, que os torna mais sensíveis ao ataque de patógenos, como demonstraram Wells \& Uota (1970). Os dados obtidos mostram que, para outros parâmetros avaliados, as respostas ao $\mathrm{CO}_{2}$ foram quadráticas,

TABELA 1 - Significância do teste F da análise da variância para o efeito dos tratamentos sobre as variáveis analisadas em caqui cv. Quioto, armazenado por 3 meses em atmosfera controlada mais 3 dias em temperatura ambiente (18-20 $\left.{ }^{\circ} \mathrm{C}\right)$. Santa Maria - RS, 1999.

\begin{tabular}{|c|c|c|c|c|c|c|}
\hline FATORES & $\begin{array}{l}\text { SST } \\
\left({ }^{\circ} \mathbf{B}\right)\end{array}$ & $\begin{array}{c}\text { Perda de peso } \\
(\%)\end{array}$ & $\begin{array}{c}\text { Podridões } \\
(\%)\end{array}$ & IEE & $\begin{array}{l}\text { Firmeza } \\
\text { de polpa }(\mathbf{N})\end{array}$ & $\begin{array}{c}\text { Cor CIE Lab } \\
\left(\mathbf{a}^{*}+\mathbf{b}^{*}\right)\end{array}$ \\
\hline Temperatura $\left({ }^{\circ} \mathrm{C}\right)$ & $\mathrm{ns}$ & $\mathrm{ns}$ & $\mathrm{ns}$ & $\mathrm{ns}$ & $* *$ & $\mathrm{~ns}$ \\
\hline $\mathrm{CO}_{2}(\mathrm{kPa})$ & $* *$ & $\mathrm{~ns}$ & $* *$ & $* *$ & $\mathrm{~ns}$ & $* *$ \\
\hline Temp. $\mathrm{X} \mathrm{CO}_{2}$ & $\mathrm{~ns}$ & $\mathrm{~ns}$ & $\mathrm{~ns}$ & $\mathrm{~ns}$ & $\mathrm{~ns}$ & $\mathrm{~ns}$ \\
\hline$\overline{\mathrm{CV}(\%)}$ & 0,98 & 22,89 & 28,51 & 13,20 & 21,94 & 2,79 \\
\hline
\end{tabular}

ns = não significativo; $* *=$ significativo pelo teste $\mathrm{F}(0,05)$.

SST = Sólidos solúveis totais; IEE = Índice de escurecimento da epiderme. 
TABELA 2 - Efeito da temperatura de armazenamento na conservação de caqui cv. Quioto mantido a 0; 5 e 10kPa de $\mathrm{CO}_{2}$ por 3 meses em atmosfera controlada mais 3 dias em temperatura ambiente (18-20 $)$. Santa Maria - RS, 1999.

\begin{tabular}{llccccc}
\hline $\begin{array}{c}\text { Temperatura } \\
\left({ }^{\circ} \mathbf{C}\right)\end{array}$ & $\begin{array}{c}\text { SST } \\
\left({ }^{\circ} \mathbf{B}\right)\end{array}$ & $\begin{array}{c}\text { Perda de peso } \\
(\boldsymbol{\%})\end{array}$ & $\begin{array}{c}\text { Podridões } \\
(\boldsymbol{\%})\end{array}$ & IEE & $\begin{array}{c}\text { Firmeza } \\
\text { de polpa }(\mathbf{N})\end{array}$ & $\begin{array}{c}\text { Cor CIE Lab } \\
\left(\mathbf{a}^{*}+\mathbf{b}^{*}\right)\end{array}$ \\
\hline $\mathbf{- 1 , 0}$ & $16,0^{\text {ns }}$ & $2,27^{\text {ns }}$ & $61,6^{\text {ns }}$ & $1,95^{\text {ns }}$ & $28,9^{* *}$ & $81,3^{\text {ns }}$ \\
$\mathbf{- 0 , 5}$ & 15,9 & 2,03 & 43,7 & 1,90 & 23,7 & 81,1 \\
Média & 16,0 & 2,15 & 52,6 & 1,93 & 26,3 & 81,1 \\
\hline $\mathbf{C V}(\%)$ & 0,98 & 22,89 & 28,51 & 13,20 & 21,94 & 2,79 \\
\hline
\end{tabular}

ns não significativo na vertical. $* *$ diferença entre as médias pelo teste $\mathrm{F}(0,05)$

SST = Sólidos solúveis totais; IEE = Índice de escurecimento da epiderme.
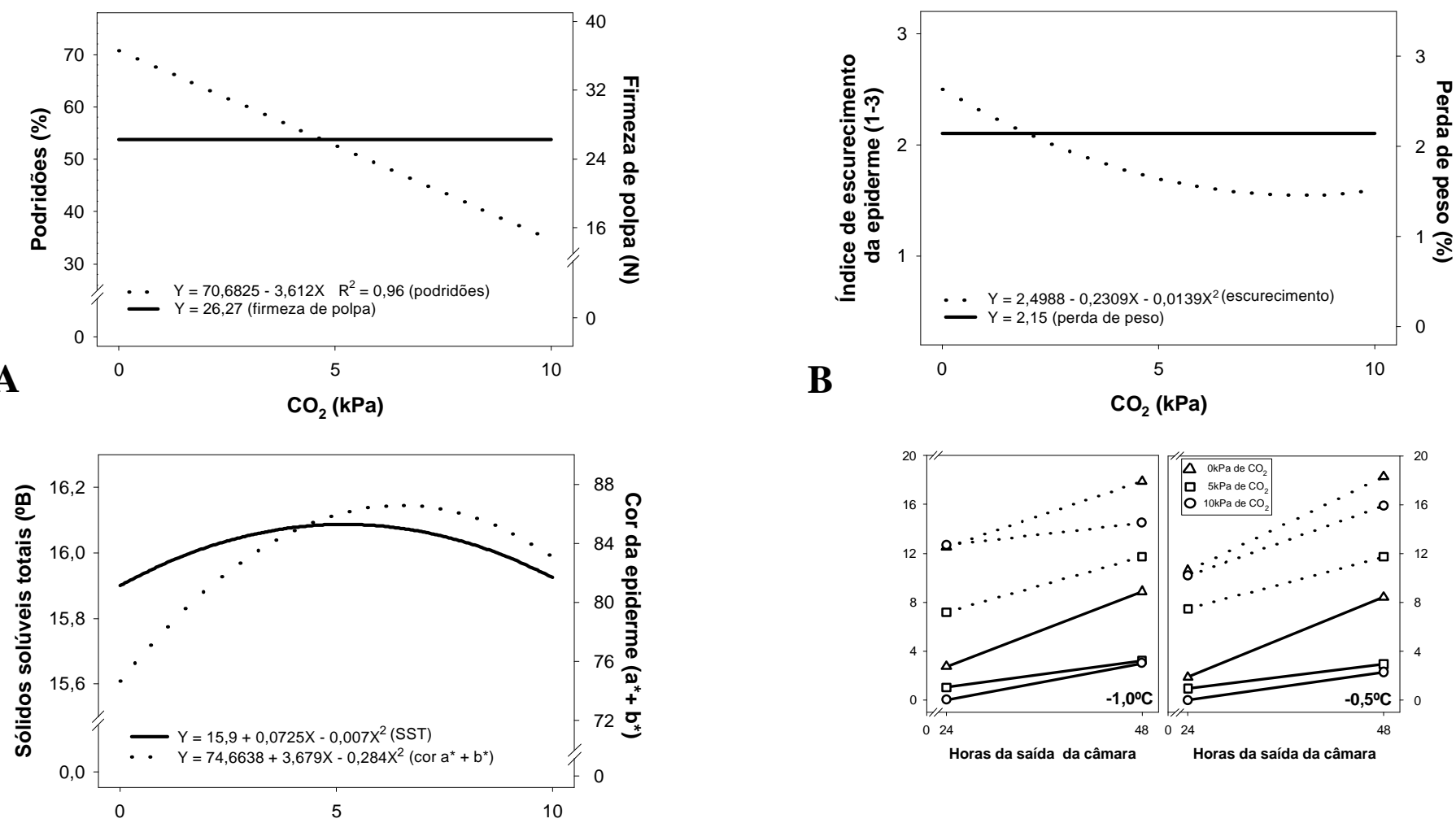

C $\mathrm{CO}_{2}(\mathrm{kPa})$

D

FIGURA 1 - Incidência de podridões e firmeza de polpa (A), índice de escurecimento de epiderme e perda de peso (B), sólidos solúveis totais e cor da epiderme (C) e evolução da produção de etileno $\left(\mu \mathrm{LC}_{2} \mathrm{H}_{4} \cdot \mathrm{kg}^{-1} \cdot \mathrm{h}^{-1}\right.$ - linhas cheias) e da respiração $\left(\mathrm{mLCO}_{2} \cdot \mathrm{kg}\right.$ ${ }^{1} \cdot \mathrm{h}^{-1}$ - linhas tracejadas) (D) de caqui cv. Quioto, em função da pressão parcial de $\mathrm{CO}_{2}$ durante o armazenamento por 3 meses em atmosfera controlada, nas temperaturas de $-1,0^{\circ} \mathrm{Ce}-0,5^{\circ} \mathrm{C}$, mais 3 dias na temperatura de $18-20^{\circ} \mathrm{C}$. Santa Maria - RS, 1999.

indicando que o ponto de máxima eficiência técnica está abaixo de 10kPa. Portanto, elevando os teores deste gás para concentrações muito acima das estudadas, podemos ultrapassar o limite para a manifestação de dano fisiológico. No entanto, pressões parciais acima de $15 \mathrm{kPa}$ já foram estudadas para a cultivar Fuyu, sendo eficiente no controle de podridões e não causando dano fisiológico na forma de escurecimento de epiderme (Brackmann et al., 1997). Assim sendo, em virtude da alta incidência de prodridões verificada, aproximadamente $35 \%$ com $10 \mathrm{kPa}$ de $\mathrm{CO}_{2}$, e da eficiência do $\mathrm{CO}_{2}$ no controle de fungos, é provável que pressões parciais de $\mathrm{CO}_{2}$ ainda mais elevadas na temperatura de $-1,0^{\circ} \mathrm{C}$ possam ter um melhor desempenho.

\section{CONCLUSÃO}

A temperatura de $-1,0^{\circ} \mathrm{C}$, pela redução da perda de firmeza de polpa, e pressões parciais de $\mathrm{CO}_{2}$ próximas a $10 \mathrm{kPa}$, pela redução de podridões e escurecimento de epiderme, é melhor condição de armazenamento dentre as estudadas para o caqui cv. Quioto. Nesta condição, o período de armazenamento deve ser inferior a 3 meses, devido às altas perdas por podridões, ficando, no entanto, com firmeza de polpa acima da considerada mínima para o consumo. 


\section{REFERÊNCIAS BIBLIOGRÁFICAS}

BEN-ARIE, R.; ZUTKHI, Y. Extending the storage life of 'Fuyu' persimmon by modified atmosphere packaging. Hortscience, Alexandria, v.27, n.7, p.811-813, 1992.

BRACKMANN, A.; STEFFENS, C.A.; MAZARO, S.M. Armazenamento de caqui (Diospyrus kaki, L.), cv. Fuyu, em condições de atmosfera modificada e controlada. Revista Brasileira de Armazenamento, Viçosa, v.24, n.2, p.42-46, 1999.

BRACKMANN, A.; CHITARRA, A.B. Atmosfera controlada e atmosfera modificada. In: BORÉM, F.M. Armazenamento e processamento de produtos agrícolas Lavras : UFLA/SBEA, 1998. 282 p.

BRACKMANN, A.; DONAZZOLO, J. Armazenagem de caqui. In: ENCONTRO NACIONAL SOBRE FRUTICULTURA DECLIMA TEMPERADO, 3., 2000, Fraiburgo.Anais... Videira:EPAGRI, 2000. p.99-102.

BRACKMANN, A.; MAZARO, S.M.; SAQUET, A.A. Frigoconservação de caquis (Diospyrus kaki, L.) das cultivares Fuyu e Rama Forte. Ciência Rural, Santa Maria, v.27, n.4, p.561$565,1997$.

BRACKMANN, A.; SAQUET, A.A. Efeito da temperatura e condições de atmosfera controlada sobre a conservação de caqui (Diospyrus kaki, L.). Ciência Rural, Santa Maria, v.25, n.2, p.215218, 1995.

BURMEISTER, D.M.; BALL, S.; GREEN, S. et al. Interaction of hot water treatments and controlled atmosphere storage on quality of 'Fuyu' persimmons. Postharvest Biology and Technology, Amsterdam, v.12, n.1, p.71-81, 1997.

GUELFAT-REICH, S.; BEN-ARIE, R.; METAL, N. Effects of $\mathrm{CO}_{2}$ during and following storage on removal of astringent and keeping quality of 'Triumph' persimmons. Journal of the American Society for Horticultural Science, Alexandria, v.100, n.2, p.9598, 1975.
KADER, A.A. Biochemical and physiological basis for effects of controlled and modified atmospheres on fruits and vegetables. Food Technology, Chicago, v.40, n.5, p.99-104, 1986.

KIM, Y.S.; JEONG, S.B.; SON, D.S.; et al. Studies of the causal factors of skin browning during storage and its control in nonastringent persimmon. Postharvest News and Information, Wallingford, v.3, n.4, p.190, 1992.

LEE, S.K.; SHIN, I.S.; PARK, Y.M. Factors involved in skin browning of non-astringent 'Fuyu' persimmon. Acta Horticulturae, Wageningen, n.343, p.300-303, 1993.

MARTINS, F.P.; PEREIRA, F.M. Cultura do caquizeiro. Jaboticabal : FUNEP, 1989. 71p.

PARK, Y.S. Changes in fruit skin blackening, phenolic acids and ethanol production of non-astringent 'Fuyu' persimmon fruits during CA storage. In: INTERNATIONAL CONTROLLED ATMOSPHERE RESEARCH CONFERENCE, 7., 1997, Davis. Proceedings... Davis : University of California, 1997. v.3, p.170-176.

PRUSKY, D.; PEREZ, A.; ZUTKHI, Y.; et al. Effect of modified atmosphere for control of black spot, caused by Alternaria alternata, on stored persimmon fruits. Phytopathology, Palo Alto, v.87, n.2, p.203-208, 1997.

TURK, R. The cold storage of persimmons (Diospyros kaki cv. fuyo) harvested at different maturities and the effect of different $\mathrm{CO}_{2}$ applications on fruit ripening. Acta Horticulturae, Wageningen, n.343, p.190-194, 1993.

WELLS, J.M.; UOTA, M. Germination and growth of five fungi in low-oxygen and high-carbon dioxide. Phytopathology, Palo Alto, v.60. n.1, p.50-53, 1970.

WRIGHT, K.P.; KADER, A.A. Effect of controlled-atmosphere storage on the quality and carotenoid content of sliced persimmons and peaches. Postharvest Biology and Technology, Amsterdam, v.10, n.1, p.89-97, 1997. 